\title{
Percutaneous direct thrombin injection with hydrodissection to manage type II endoleak after endovascular abdominal aortic aneurysm repair
}

\author{
Bartosz Żabicki ${ }^{1 A, B, D, E}$, Kinga Kubiak ${ }^{1 B, D, E, F}$, Marcin Gabriel|2B,D ${ }^{2 B}$ Robert Juszkat ${ }^{1 B, D}$ \\ 'Department of General and Interventional Radiology, Poznan University of Medical Sciences, Poznan, Poland \\ 2Department of General and Vascular Surgery, Poznan University of Medical Sciences, Poznan, Poland
}

\begin{abstract}
Type II endoleak is the most common complication after endovascular abdominal aortic aneurysm repair (EVAR). The management remains controversial. We present a case in which endoleak was successfully treated by direct percutaneous thrombin injection with hydrodissection. This method seems to be a safe and feasible alternative method for treatment of type II endoleak.
\end{abstract}

Key words: endoleak, thrombin, hydrodissection.

\section{Introduction}

Endovascular abdominal aortic aneurysm repair (EVAR) is complicated by various types of endoleak in 10-50\% of patients [1]. Type II endoleak is the most common, occurring in approximately $20 \%$ of cases [2,3]. However, management of type II endoleak remains controversial. If the endoleak is accompanied by an aneurysm expansion (>5 mm) and if it is persistent (> 6 months), an intervention should be considered [2]. A case in which the endoleak was successfully treated by direct percutaneous thrombin injection is presented in the article. Hydrodissection in the retroperitoneal space was utilised to form safe and sufficient access path to the aorta. To our knowledge, there is no published report regarding this accessory technique in percutaneous endoleak management.

\section{Case report}

An 86-year-old male patient was treated with EVAR due to a $62-\mathrm{mm}$ infrarenal aortic aneurysm. The patient suffered from coronary artery disease, arterial hypertension, general atherosclerosis, and benign prostatic hyperplasia accompanied by urolithiasis. Zenith stent-graft (Cook, Denmark) with suprarenal fixation was chosen to treat the aneurysm. Common iliac arteries were widened bilaterally, and therefore distal landing zones of the graft were set to the external iliac arteries. Control angiography following the procedure revealed no endoleak. Post-operative recovery was uneventful.

A routine follow-up computed tomography (CT) scan performed one month post-operatively showed a significant type II endoleak supplied by the inferior mesenteric artery. It did not thrombose until the follow-up CT performed after six months. Subsequent imaging at one year depicted persistent endoleak with aneurysm sack enlargement to $68 \mathrm{~mm}$.

The patient was re-admitted to the Department of Vascular Surgery in order to treat the persisting endoleak. The patient was initially scheduled to the Interventional Radiology Angio Suite for embolisation. Selective catheterisation of the superior mesenteric artery was achieved from the femoral access, but further superselective approach to the origin of inferior mesenteric artery through

Correspondence address:

Dr. Kinga Kubiak, Department of General and Interventional Radiology, Poznan University of Medical Sciences, 1/2 Długa St., 61-848 Poznań, Poland,

e-mail:kinga_kubiak@yahoo.com

Authors' contribution:

A Study design · B Data collection · C Statistical analysis · D Data interpretation · E Manuscript preparation · F Literature search · G Funds collection 


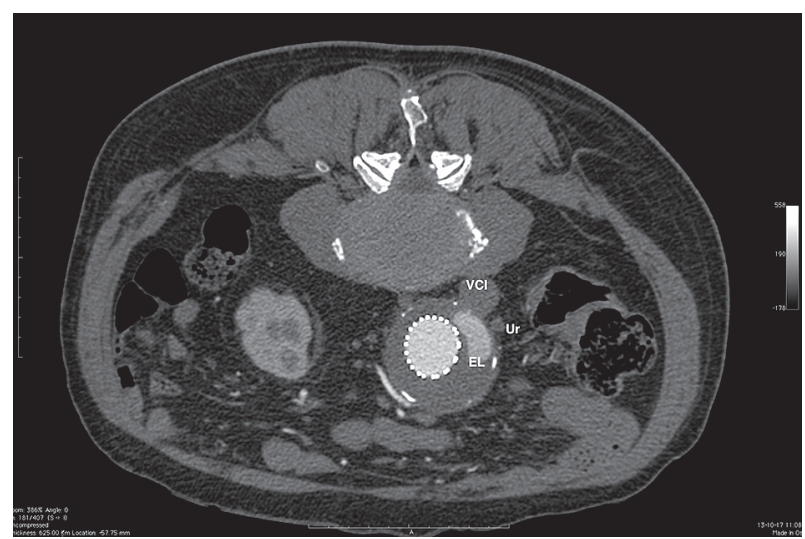

Figure 1. Axial computed tomography in prone position - endoleak type 2 right-sided to the endograft ( $\mathrm{EL}$ - endoleak, $\mathrm{Ur}$ - ureter, $\mathrm{VCl}$ - vena cava inferior)
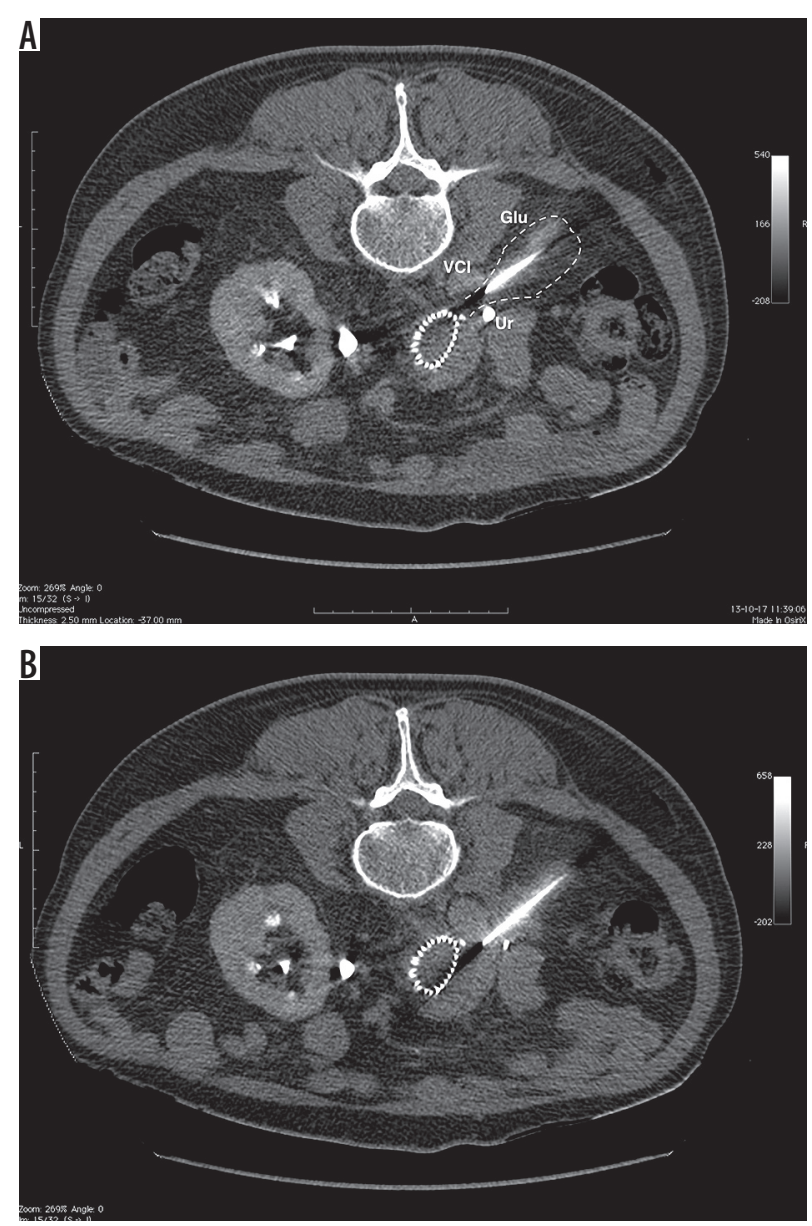

Figure 2. A) Needle advanced close to vena cava inferior and the ureter the space between the structures sufficiently splayed with an injection of $5 \%$ glucose in water (Glu - glucose in water, $\mathrm{Ur}$ - ureter, $\mathrm{VCl}$ - vena cava inferior). B) Needle tip in the endoleak zone, shortly before thrombin injection

the arc of Riolan was impossible. It was not possible to pass the vascular tortuosities to enable embolisation of the aneurysm sack and the origin of the inferior mesenteric artery (IMA) with the use of any microcatheters available in the angio suite (including neuromicrocath-

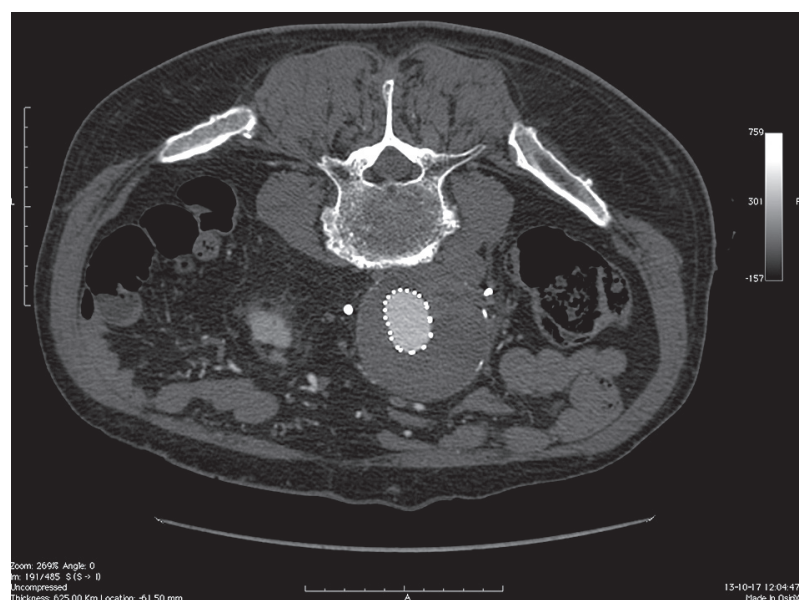

Figure 3. Control computed tomography after thrombin injection and needle removal- no persistent endoleak, no haemorrhagic complications, and no ureter injury

eters). Due to unsuccessful endovascular procedure, direct puncture of the aneurysm sack was considered. The patient was scheduled to CT, settled in a prone position, and conscious sedation was administered. Initial scan with $60 \mathrm{ml}$ of contrast agent in arterial and delayed phase was performed to re-evaluate the procedure planning process and the puncture site. Endoleak zone within the aneurysm sack was located right to the stent-graft; therefore, direct right retroperitoneal puncture was the safest and the only option to access the sack of the aneurysm (Figure 1). A 20-cm 18G biopsy needle was advanced beneath the lower pole of the right kidney and stopped at a short distance from the vena cava inferior. CT-fluoroscopy was not available, so the needle was guided in a "scan and go" manner. After placing the tip of the needle in the area between ureter and vena cava inferior, approximately $20 \mathrm{ml}$ of $5 \%$ glucose was injected to achieve so-called hydrodissection in the retroperitoneal space in order to provide sufficient space for safe access to the aorta (Figure 2A). The needle was advanced gently to the aneurysm lumen until the backflow in the needle hub confirmed its correct position (Figure 2B). Subsequently, three ampules (1200 Units) of thrombin (Biothrombin, Poland) were injected to the lumen of the aneurysm. The needle was then retrieved. Post-procedural scan with $60 \mathrm{ml}$ of contrast agent in arterial and delayed phase confirmed no signs of endoleak and no complications such as bleeding or damage of the vena cava inferior or ureter (Figure 3). Follow-up contrast-enhanced ultrasound (CEUS) at the end of hospital stay and at one month and six months confirmed no persistent endoleak (Figure 4). Aneurysm diameter at six months measured $66 \mathrm{~mm}$.

\section{Discussion}

EVAR has evolved in recent decades. Nowadays, it is a commonly performed procedure used to treat abdominal aortic aneurysm because it constitutes a minimally 
invasive alternative to open repair. It is associated with a low rate of short-term and mid-term complications, although a long-term prognosis of EVAR is uncertain, mainly because of the endoleaks that represent a common complication after EVAR. By definition, the term "endoleak" denotes the persistence of blood flow outside the lumen of the graft but within the aneurysm sac $[1,4,5]$.

Endoleaks can be classified into different types according to the time of development (acute and late) and the origin (types I-V). It is documented that types I and III are related with high risk of rupture [1], so urgent treatment is required. However, management of type II endoleaks remains controversial. This type of endoleak is, by definition, associated with retrograde flow into the aneurysm sac from arterial branches, commonly the inferior mesenteric or lumbar arteries [6]. Some researchers advocate intervention in all cases, while others choose a conservative approach and believe that intervention is required only if the endoleak is accompanied by aneurysm expansion (>5 mm) or it is persistent (> 6 months) [2]. According to the literature, up to 54\% [4] of type II endoleaks resolve spontaneously. However, not all type II endoleaks are benign; some of them can be associated with complications as aneurysm sac enlargement or even rupture [6].

Several options for the treatment are described in the literature, including transarterial approach or direct puncture. Transarterial embolisation is the most commonly used approach, although there is evidence suggesting that the translumbar approach provides more stable results in the long-term [3] and enables shorter fluoroscopy and procedure time [7]. However, the proper management has to be adapted individually according to the location of the endoleak and the patient's anatomy. In our case, anatomy made the transarterial approach impossible, so the translumbar approach was an effective alternative method.

Direct puncture of the endoleak can be performed using translumbar or transabdominal approach depending on the location of the endoleak. Direct percutaneous translumbar puncture and embolisation of left sided endoleaks have been described in literature [8]. A relatively large number of potential complications, including retroperitoneal haemorrhage, inadvertent intracaval coil migration, or aortocaval fistula, makes the treatment of right-sided endoleaks more difficult. It is associated with the fact that the operator has to traverse the vena cava

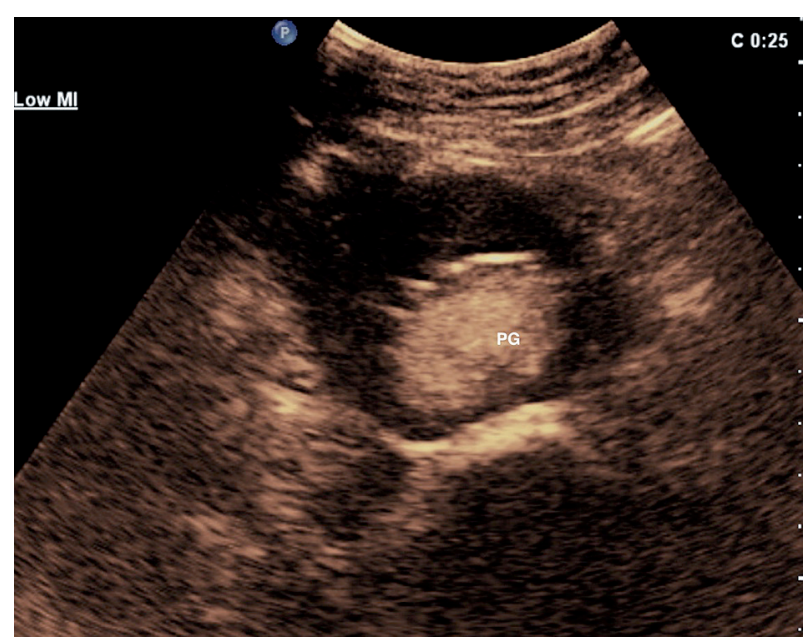

Figure 4. Follow-up contrast-enhanced ultrasound at six months - stentgraft patent (PG), no endoleak visible

inferior. In the case described, the operator's aim was to avoid a transcaval approach, so percutaneous injection of glucose with retroperitoneal hydrodissection of anatomical structures was performed.

As reported in the literature, so-called hydrodissection plays a significant role in avoiding the potential damage of adjacent structures in procedures such as ablation or biopsy [9]. Depending on the procedure, $5 \%$ dextrose in water (D5W) or saline is used to promote safe needle access or to minimise potential damage of adherent organs. In our case hydrodissection was used to splay the space between the vena cava inferior and right ureter, which enabled safe access to the endoleak zone and its treatment by thrombin injection. Ultrasound-guided percutaneous thrombin injection is commonly used for treatment of postcatheterisation pseudoaneurysm because the therapy is quick, safe, well-tolerated, and cost-effective [10].

\section{Conclusions}

In the presented case, percutaneous direct thrombin injection with hydrodissection seemed a safe and feasible alternative method for treatment of type II endoleak.

\section{Conflict of interest}

The authors declare that they have no conflict of interest.

\section{References}

1. Rand T, Uberoi R, Cil B, et al. Quality Improvement Guidelines for Imaging Detection and Treatment of Endoleaks following Endovascular Aneurysm Repair (EVAR). Cardiovasc Intervent Radiol 2013; 36: 35-45.

2. Steinmetz E, Rubin BG, Sanchez LA, et al. Type II endoleak after endovascular abdominal aortic aneurysm repair: A conservative approach with selective intervention is safe and cost-effective. J Vasc Surg 2004; 39: 306-313.

3. Baum RA, Carpenter JP, Golden MA, et al. Treatment of type 2 endoleaks after endovascular repair of abdominal aortic aneurysm: Comparison of transarterial and translumbar techniques. J Vasc Surg 2002; 35: 23-29. 
4. Sidloff DA, Stather PW, Choke E, et al. Type II endoleak after endovascular aneurysm repair. Br J Surg 2013; 100: 1262-1270.

5. White GH, Weiyun Y, May J, et al. Endoleak as a Complication of Endoluminal Grafting of Abdominal Aortic Aneurysm: Classification, Incidence, Diagnosis and Management. J Endovasc Surg 1997; 4: 152-168.

6. Stavropoulos SW, Park J, Fairman R, et al. Type 2 Endoleak Embolization Comparison: Translumbar Embolization versus Modified Transarterial Embolization. J Vasc Interv Radiol 2009; 20: 1299-1302.

7. Yang RY, Tan KT, Beecroft JR, et al. Direct sac puncture versus transarterial embolization of type II endoleaks: An evaluation and comparison of outcomes. Vascular 2016; 25: 1-7.
8. van den Berg JC, Nolthenius RP, Casparie JW, et al. CT-guided thrombin injection into aneurysm sac in a patient with endoleak after endovascular abdominal aortic aneurysm repair. AJR 2000; 175: 1649-1651.

9. Asvadi NH, Arellano RS. Hydrodissection-Assisted Image-Guided Percutaneous Biopsy of Abdominal and Pelvic Lesions: Experience With Seven Patients. AJR 2015; 204: 865-867.

10. La Perna L, Olin JW, Goines D, et al. Ultrasound-Guided Thrombin Injection for the Treatment of Postcatheterization Pseudoaneurysms. Circulation 2000; 102: 2391-2395. 